\title{
Article \\ Comprehensive Analysis of Chemotherapeutic Agents That Induce Infectious Neutropenia
}

\author{
Mashiro Okunaka ${ }^{1,2}$, Daisuke Kano ${ }^{2}$, Reiko Matsui ${ }^{2}$, Toshikatsu Kawasaki ${ }^{2}$ and Yoshihiro Uesawa ${ }^{1, *(1)}$ \\ 1 Department of Medical Molecular Informatics, Meiji Pharmaceutical University, Kiyose, \\ Tokyo 204-8588, Japan; mokunaka@east.ncc.go.jp \\ 2 Department of Pharmacy, National Cancer Center Hospital East, Kashiwa, Chiba 277-8577, Japan; \\ dkano@east.ncc.go.jp (D.K.); rmatsui@east.ncc.go.jp (R.M.); tkawasak@east.ncc.go.jp (T.K.) \\ * Correspondence: uesawa@my-pharm.ac.jp; Tel.: +81-42-495-8983
}

Citation: Okunaka, M.; Kano, D.;

Matsui, R.; Kawasaki, T.; Uesawa, Y.

Comprehensive Analysis of

Chemotherapeutic Agents That

Induce Infectious Neutropenia.

Pharmaceuticals 2021, 14, 681.

https://doi.org/10.3390/ph14070681

Academic Editor: Dinender K. Singla

Received: 8 June 2021

Accepted: 12 July 2021

Published: 15 July 2021

Publisher's Note: MDPI stays neutral with regard to jurisdictional claims in published maps and institutional affiliations.

Copyright: (c) 2021 by the authors. Licensee MDPI, Basel, Switzerland. This article is an open access article distributed under the terms and conditions of the Creative Commons Attribution (CC BY) license (https:// creativecommons.org/licenses/by/ $4.0 /)$.

\begin{abstract}
Chemotherapy-induced neutropenia (CIN) has been associated with a risk of infections and chemotherapy dose reductions and delays. The chemotherapy regimen remains one of the primary determinants of the risk of neutropenia, with some regimens being more myelotoxic than others. Although a number of clinical trials have currently highlighted the risk of CIN with each chemotherapy regimen, only a few ones have comprehensively examined the risk associated with all chemotherapeutic agents. Therefore, this study aimed to investigate the risk factors and characteristics of CIN caused by each neoplastic agent using data from the large voluntary reporting Food and Drug Administration Adverse Event Reporting System database. Initially, univariate analysis showed that an age $\geq 65$ years, the female sex, and treatment with chemotherapeutic agents were factors that caused CIN. Then, cluster and component analyses showed that cytotoxic agents (i.e., alkylating agents, antimetabolic agents, antineoplastic antibiotics, platinating agents, and plant-derived alkaloids) were associated with infection following neutropenia. This comprehensive analysis comparing CIN risk suggests that elderly or underweight patients treated with cytotoxic drugs require particularly careful monitoring.
\end{abstract}

Keywords: chemotherapy-induced neutropenia; chemotherapeutic agent; cancer

\section{Introduction}

Neutrophils generally comprise approximately half to two-thirds of all white blood cells (immune cells) and protect against bacterial infections [1]. Patients who develop neutropenia may have a higher-than-normal risk of infections, and the severity of subsequent infections is also higher. Chemotherapeutic agents act on the bone marrow, where active cell division occurs, and deplete hematopoietic stem cells [2], leading to a decreased circulating absolute neutrophil count [3]. Patients receiving chemotherapy have been reported to experience a temporary reduction in their neutrophil counts [4,5]. Chemotherapy-induced neutropenia (CIN) remains a common dose-limiting toxicity for chemotherapeutic agents, causing treatment delays and/or dose reductions [6].

Furthermore, CIN increases in severity as the absolute neutrophil count declines below $500 / \mu \mathrm{L}$ [5]. Febrile neutropenia refers to the occurrence of fever during a period of severe neutropenia.

Although a number of clinical trials have investigated the risk of CIN across multiple chemotherapy regimens, comparing their results based on indications remains challenging. Furthermore, triplet/doublet regimens promote a higher CIN incidence and myelotoxicity than single-agent regimens [7]. A retrospective comparative study on the toxicity of multiple chemotherapeutic agents frequently observed febrile neutropenia with paclitaxel for breast cancer $(18 \%)$, carboplatin plus paclitaxel for lung cancer $(23 \%)$, and oxaliplatin and fluorouracil plus leucovorin for colorectal cancer (23\%) [8]. However, only a few studies 
have comprehensively examined the risk associated with multiple chemotherapeutic agents, and to the best of our knowledge, none have comprehensively investigated all agents.

The US Food and Drug Administration (FDA) publishes the FDA Adverse Event Reporting System (FAERS) database. This is a voluntary reporting system database for the postmarketing surveillance of all approved drugs and therapeutic biologics, available to public and scientists from the FDA's home page $[9,10]$. The present study comprehensively analyzed all chemotherapeutic agents available in the FAERS database and compared CIN risk and detailed characteristics.

\section{Results}

\subsection{Data Presentation}

Among the 35,393,413 rows (21,349 categories) of adverse events registered in the FAERS, 121,722 rows (eight categories) were related to neutropenia. The drug/biologic information for any medication as associated with an event (DRUG), MedDRA terms coded for adverse events (REAC), and patient demographic and administrative information (DEMO) tables included 35,393,413, 12,991,342, and 2,094,270 rows, respectively. The total number of rows, as shown in the data analysis table, was 9,131,876 (Figure 1).

\begin{tabular}{|c|c|}
\hline Deduplicated REAC & \\
\hline $\begin{array}{l}\text { - } \text { Primary ID } \\
\text { - } \text { Drug name } \\
\text { - } \text { Code for drug's reported role in } \\
\text { event (selected "primary suspect }\end{array}$ & Deduplicated DRUG \\
\hline $\begin{array}{l}\text { drug" only) } \\
\text { - ATC code } \\
\end{array}$ & $\begin{array}{l}\text { Primary ID } \\
\text { - Adverse event Preferred Term } \\
\qquad 35,393,413 \text { rows }\end{array}$ \\
\hline Deduplicated DEMO & \\
\hline - Primary ID & Data Analysis Table \\
\hline $\begin{array}{l}\text { - Gender code } \\
\text { - Patient's weight } \\
\text { - Unit abbreviation for patient's } \\
\text { weight } 2,094,270 \text { rows }\end{array}$ & $\begin{array}{l}\text { Primary ID } \\
\text { - } \text { Adverse event Preferred Term } \\
\text { - } \text { Genetic name of drug } \\
\text { - } \text { Age } \\
\text { - Unit abbreviation for patient's age } \\
\text { - } \text { Gender code } \\
\text { - } \text { Patient's weight } \\
\text { - Unit abbreviation for patient's } \\
\text { weight } \quad 9,131,876 \text { rows }\end{array}$ \\
\hline
\end{tabular}

Figure 1. Flow chart for the construction of the data analysis table. The REAC table was classified into three categories: "suspected medicine", "concomitant medicine" and "interaction medicine". We extracted only "primary suspect drug" information relevant to these categories from the REAC table. Duplicate data were then removed from the REAC, DEMO, and DRUG tables. Based on the combined table, only "suspected medicine" information was used to assess the risk of diarrhea. Available information on "suspected medicine", "concomitant medicine" and "interaction medicine" was used for the time-of-onset analysis. Each table was combined, cleaned up, and then used as the data analysis table. 


\subsection{Patients Characteristics}

Significant differences in sex, age, and weight characteristics were observed in patients who developed neutropenia. Criteria for elderly patients aged $\geq 65$ years were defined based on a previous study regarding risk factors for febrile neutropenia in patients with cancer who are receiving chemotherapy [11]. More females than males showed a tendency to develop CIN (51.7\%, $n=16,751$, vs. 48.3\%, $n=15,640$; Tables 1 and 2). Patients with CIN had a median age and weight of 61 years and $68.0 \mathrm{~kg}$, respectively, whereas those without CIN had a mean age and weight of 58 years and $73.0 \mathrm{~kg}$, respectively.

Table 1. Patient characteristics.

\begin{tabular}{cccc}
\hline Characteristic & CIN & Non-CIN & $p$ Value \\
\hline Age (years) & & & \\
Median [range] & $61(0-103)$ & $58(0-110)$ & \\
$\geq 65$ years & $13,397(41.4 \%)$ & $731,626(35.9 \%)$ & $<0.0001$ \\
<65 years & $18,994(58.6 \%)$ & $1,309,160(64.1 \%)$ & \\
Sex, No & & & \\
Female & $16,751(51.7 \%)$ & $1,235,937(60.6 \%)$ & $<0.0001$ \\
Male & $15,640(48.3 \%)$ & $804,849(39.4 \%)$ & $<0.0001$ \\
Weight (kg) & & & \\
Median [range] & $68.0(0.0-150.0)$ & $73.0(0.0-150.0)$ & $<0.0001$ \\
Antineoplastic agents & & & \\
Yes & $19,720(60.9 \%)$ & $260,981(12.8 \%)$ & \\
No & $12,671(39.1 \%)$ & $1,779,805(87.2 \%)$ & \\
ATC classification & $1890(4.8 \%)$ & $10,279(0.3 \%)$ & \\
Alkylating agent & $4078(10.4 \%)$ & $34,168(1.2 \%)$ & \\
Antimetabolic agent & $1054(2.7 \%)$ & $5843(0.2 \%)$ & \\
Antineoplastic agent & $3534(9.0 \%)$ & $59,542(2.0 \%)$ & \\
Monoclonal antibody & $2111(5.4 \%)$ & $17,077(0.6 \%)$ & \\
Platinating agent & $2430(6.2 \%)$ & $72,130(2.4 \%)$ & \\
Protein kinase agent & $2347(6.0 \%)$ & $27,673(0.9 \%)$ & \\
Plant-derived alkaloids & $23 \%)$ & \\
\hline ATC, Anatomical Therapeutic Chemistry. CIN, Chemotherapy-induced Neutropenia. &
\end{tabular}

ATC, Anatomical Therapeutic Chemistry. CIN, Chemotherapy-induced Neutropenia.

Table 2. Odds ratio related with CIN.

\begin{tabular}{cccc}
\hline \multirow{2}{*}{ Variables } & Category & \multicolumn{2}{c}{ Univariate Analysis } \\
& & Odds Ratio (95\% CI) & $p$ Value \\
\hline Age (years) & $\geq 65$ vs. $<65$ & $1.394(1.365-1.424)$ & $<0.0001$ \\
Sex & Female vs. male & $1.434(1.403-1.466)$ & $<0.0001$ \\
Chemotherapeutic agents & Yes vs. No & $10.614(10.375-10.857)$ & $<0.0001$ \\
\hline
\end{tabular}

\subsection{CIN-Inducing Drugs}

Figure 2 presents a volcano plot demonstrating drugs suspected of causing neutropenia. As shown in the figure, drugs with positive lnORs on the $X$ axis were more frequently reported to have caused neutropenia than other adverse events. Moreover, drugs with higher values of logarithmically transformed inverse $p$ values on the $\mathrm{Y}$ axis had stronger significant differences. In other words, the drugs located in the right upper quadrant were more likely to induce medication-related neutropenia. The volcano plot showed that cytotoxic agents such as alkylating agents, antimetabolic agents, and antineoplastic antibiotics were particularly associated with the development of neutropenia. 


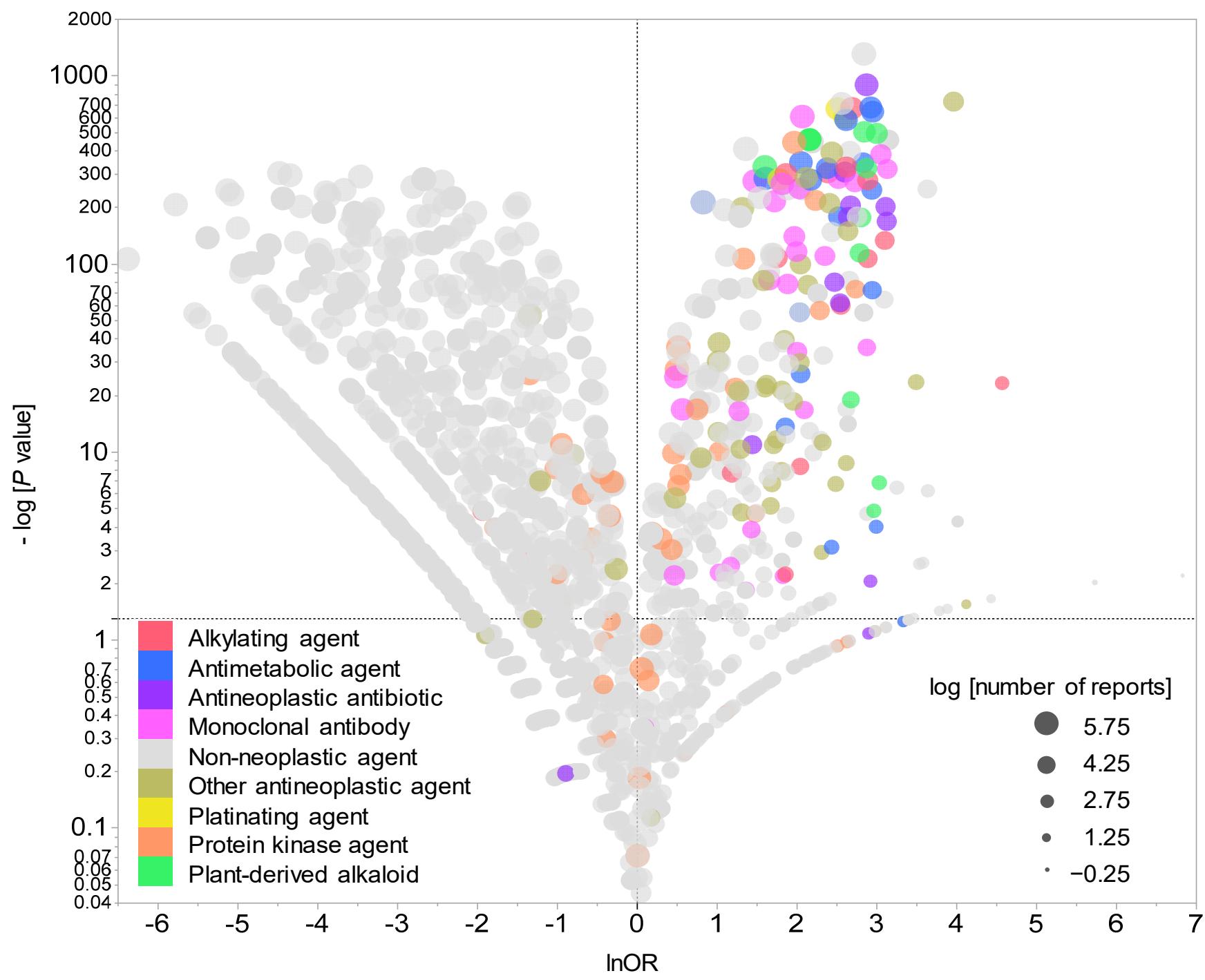

Figure 2. Drugs associated with CIN development. The $\mathrm{X}$ axis shows the natural $\ln O \mathrm{Rs}$, whereas the $\mathrm{Y}$ axis shows the common logarithm of the inverse $p$ value ( $-\log 10$ ( $p$ value)) from Fisher's exact test. The ORs were calculated using crosstabulation. The dotted line on the $\mathrm{Y}$ axis represents $p=0.05$. The plot colors indicate Anatomical Therapeutic Chemistry classification, whereas the plot size indicates the common logarithm of the total number of reported adverse events for each drug $(-0.25$ to 5.75$)$.

\subsection{Cluster Analyses of CIN-inducing Drugs}

The dendrogram generated by hierarchical cluster analysis resulted in two clusters (Figure 3), among which one was associated with neutropenia, granulocytopenia, agranulocytosis, neutropenic sepsis, neutropenic colitis, neutropenic infection, and febrile neutropenia. This cluster contained the cytotoxic agents (e.g., epirubicin, vinorelbine, and cyclophosphamide) and monoclonal antibodies (e.g., bevacizumab and trastuzumab) that are administered in combination with cytotoxic agents. Other than palbociclib which was strongly associated with neutropenia and granulocytopenia, the second cluster contained drugs that were poorly associated with these adverse events, such as protein kinase agents (e.g., regorafenib and imatinib) and immune checkpoint inhibitors (e.g., nivolumab and ipilimumab). Thus, different degrees of association with CIN were noted for each chemotherapeutic agent. 

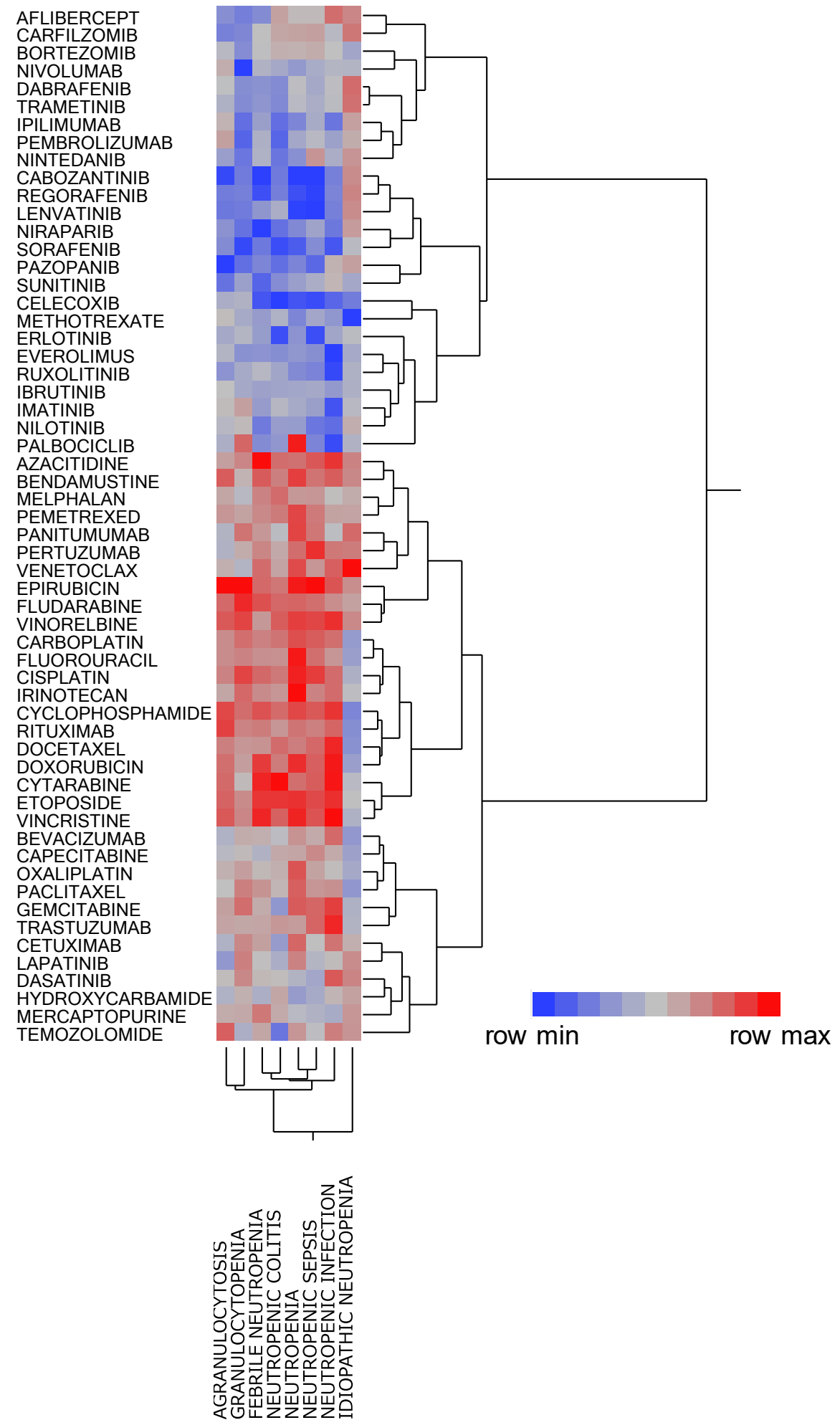

Figure 3. Classification of CIN-related chemotherapeutic agents using hierarchical cluster analysis. The dendrogram shows the relationships between 58 chemotherapeutic agents and CIN. The color map shows the load value of the principal components in red-gray-blue.

\subsection{Principal Component Analysis Related to CIN}

The contribution ratios of the principal component were $58.4 \%, 14.6 \%$, and $10.3 \%$ for the components 1, 2, and 3, respectively. Component 2 was excluded from the variable 
selection given its possible association with the number of reports (Figure 4). A scatterplot was then created using components 1 and 3 . The relationship between adverse events related to neutropenia and main components was visualized using the plot, where each adverse event was represented as a loading vector (Figure 5). The $X$ axis represents the first component, with all adverse event vectors showing a positive association. The $\mathrm{Y}$ axis represents the third component in which granulocytopenia (0.670), idiopathic neutropenia (0.383), agranulocytosis $(0.242)$, and neutropenia (0.0118) showed a positive principal component load, whereas febrile neutropenia $(-0.141)$, neutropenic colitis $(-0.179)$, and neutropenic infection $(-0.187)$, and neutropenic sepsis $(-0.288)$ showed a negative principal component load (Table 3).

Table 3. Principal component load related to CIN.

\begin{tabular}{ccc}
\hline Adverse Event & First Component & Second Component \\
\hline Granulocytopenia & 0.594 & 0.670 \\
Idiopathic neutropenia & 0.167 & 0.383 \\
Agranulocytosis & 0.801 & 0.242 \\
Neutropenia & 0.904 & 0.0118 \\
Febrile neutropenia & 0.913 & -0.141 \\
Neutropenic colitis & 0.794 & -0.179 \\
Neutropenic infection & 0.802 & -0.187 \\
Neutropenic sepsis & 0.853 & -0.288 \\
\hline
\end{tabular}

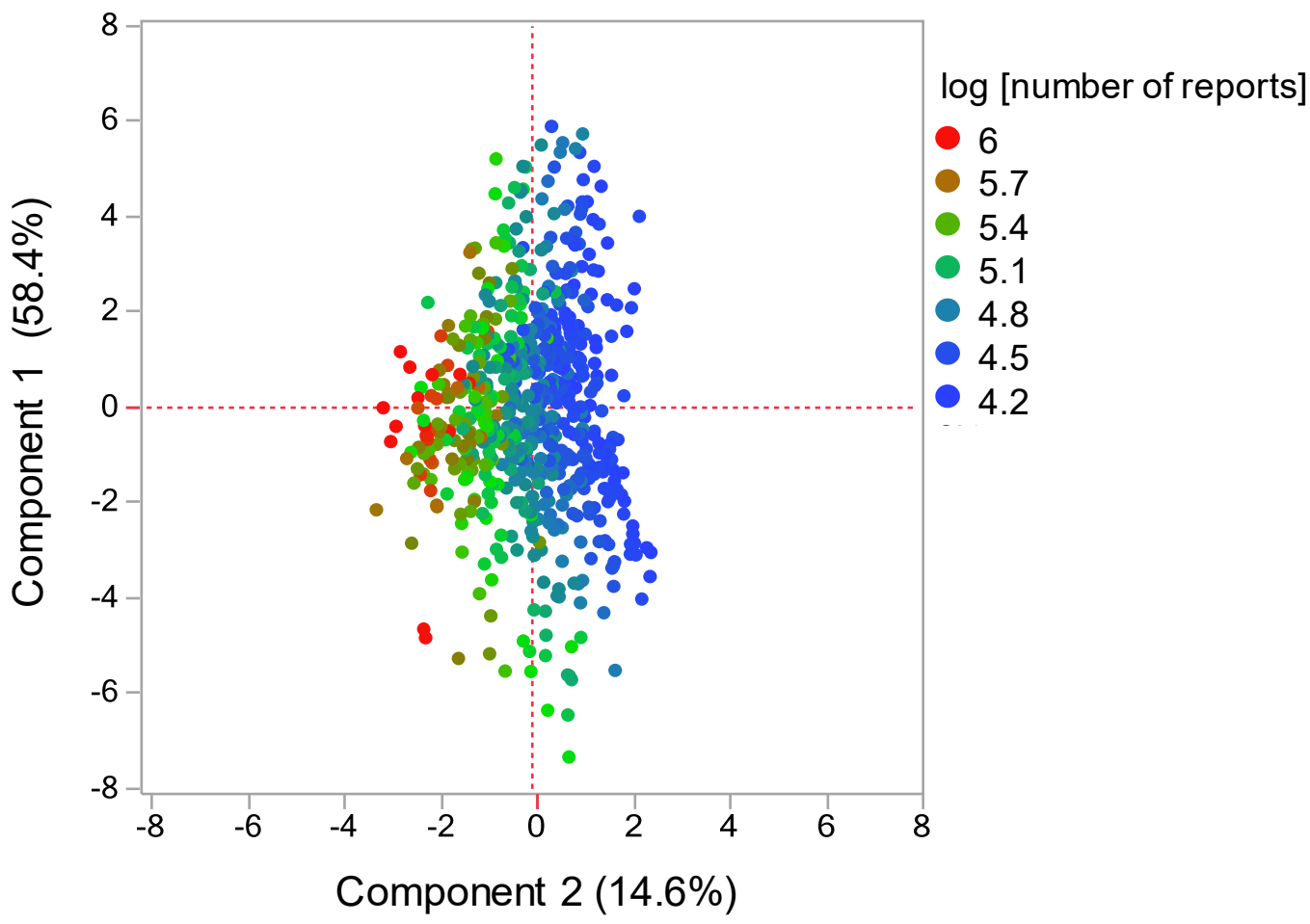

Figure 4. Score plot related with components 1 and 2. The score plot shows the relationships between the drugs and principal components. Each plot indicates a drug. Plot colors indicate log (number of reports). 


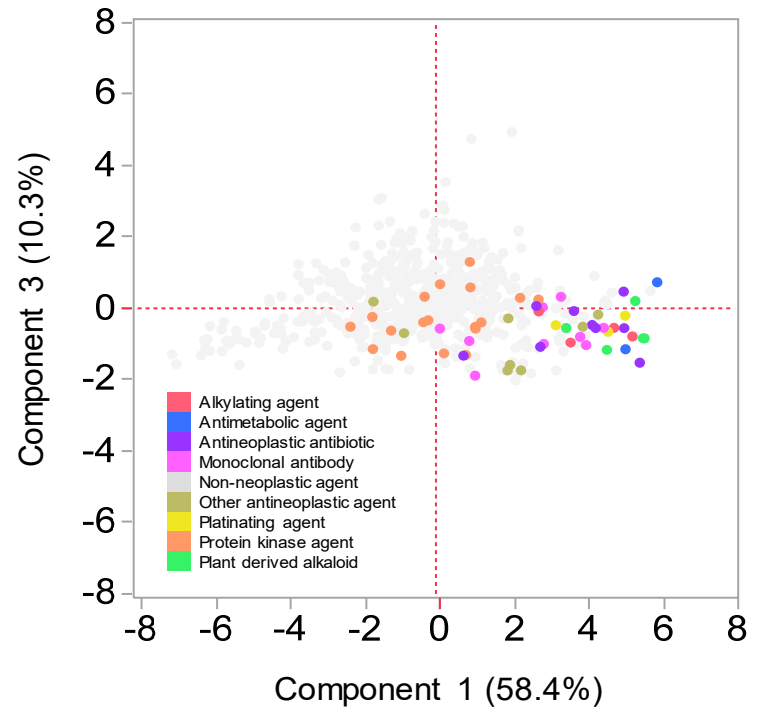

(a)

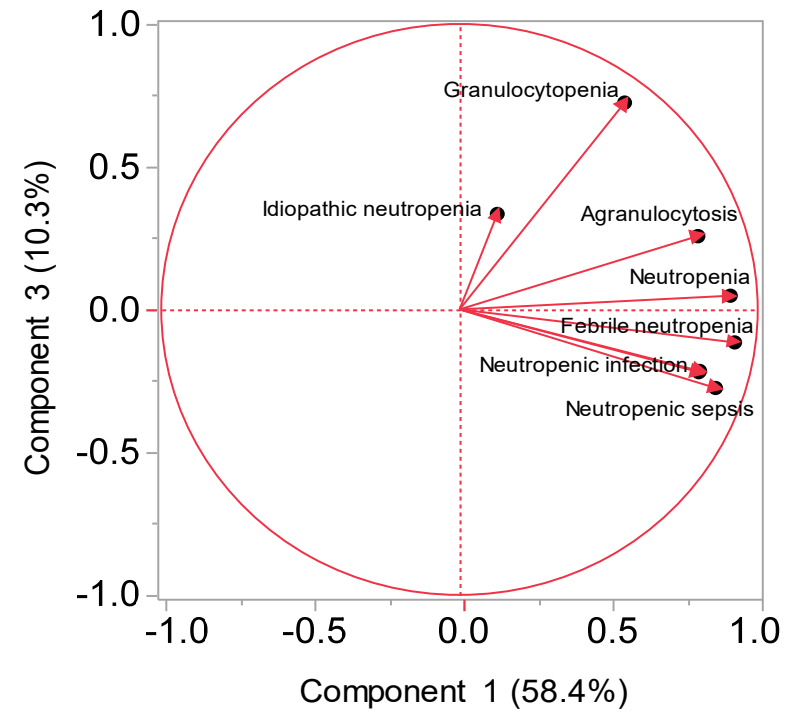

(b)

Figure 5. Relationships between CIN and drugs using principal component analysis. (a) Score plot; (b) loading vectors. (a) The score plot shows the relationships between the drugs and principal components. Each plot indicates a drug. Plot colors indicate Anatomical Therapeutic Chemistry classification. (b) Loading vectors represent the relationship between side effects and principal components. Each loading vector indicates a side effect.

\section{Discussion}

The present study examined the risk factors and characteristics of CIN and clarified the relationship between CIN and related disease. To the best of our knowledge, this is the first study to provide data on the frequency of comprehensive CIN for all drugs using the FAERS database.

Our results showed that elderly patients, those receiving antineoplastic agents, and underweight individuals (low BMI/body surface area) were more likely to develop CIN (Tables 1 and 2). In particular, alkylating agents (e.g., cyclophosphamide), plant-derived alkaloids (e.g., docetaxel), antineoplastic antibiotics (e.g., doxorubicin), and platinating agents (e.g., cisplatin) have been found to be myelosuppressive. Although it was difficult to calculate BMI because the FAERS database does not contain height information, the findings presented in this study, with the exception of sex, showed a tendency to be consistent with those reported in a previous study regarding risk factors for febrile neutropenia among patients with cancer who were receiving chemotherapy [11]. In other words, patients who present with CIN risk factors should also be vigilant regarding the development of febrile neutropenia following myelosuppression.

This study also investigated the relationship between CIN and chemotherapeutic agents. Cluster analysis, a method for classifying data into similar groups (clusters) [12], revealed that neutropenia, granulocytopenia, agranulocytosis, neutropenic sepsis, neutropenic colitis, neutropenic infection, and febrile neutropenia shared similar features (Figure 3). The present study classified 48 drugs into two clusters before evaluating the cluster characteristics. Notably, one cluster showed a strong positive association with neutropenia, granulocytopenia, agranulocytosis, neutropenic sepsis, neutropenic colitis, neutropenic infection, and febrile neutropenia, whereas the other, comprising protein kinase inhibitors and monoclonal antibodies (excluding the antimetabolite methotrexate), was less associated with CIN.

Our findings showed that palbociclib, a protein kinase inhibitor, was strongly associated with neutropenia and granulocytopenia but weakly associated with febrile neutropenia, neutropenic colitis, neutropenic sepsis, and neutropenic infection, with asymptomatic neutropenia being considered the main adverse effect of palbociclib. The PALOMA-3 trial 
showed that although $58-92 \%$ of patients had $\geq$ grade 3 neutropenia, no complications of infection and increase in febrile neutropenia had occurred [13]. Palbociclib has been reported to exert its antitumor effects by inhibiting CDK4/6 [14], which is significant owing to evidence showing that the mechanism for neutropenia development may differ from that due to cytotoxic chemotherapy.

Although cluster analysis classifies drugs with varying characteristics based on the index of similarity, comprehensively comparing the bias of explanatory variables of drugs remains difficult. Principal component analysis has been used as a method for visualizing the bias of explanatory variables by converting them into a summary index (principal component) [15]. Each adverse event and drug used in the present study was interpreted based on principal components, whereas the main component was interpreted using loading vectors that represent adverse events (Figures 4 and 5). Given that all adverse event vectors were positively associated with component 1 , such a component can be considered a comprehensive index of CIN-related side effects. Adverse event vectors were classified into those with positive and negative relationships with components 2 and 3. In component 2 , the positive adverse events included idiopathic neutropenia, neutropenic infection, and neutropenic colitis, whereas negative adverse events included febrile neutropenia, neutropenic sepsis, neutropenia, agranulocytosis, and granulocytopenia. However, Figure 4 shows that the positive-negative relationship in component 2 was associated with the number of reports. In component 3 , the positive adverse events included granulocytopenia, idiopathic neutropenia, agranulocytosis, and neutropenia (Figure 5), whereas the negative adverse events included febrile neutropenia, neutropenic colitis, and neutropenic sepsis, which have been considered to affect bacterial infection. Therefore, component 3 can be considered a comprehensive indicator of infections following myelosuppression.

Drugs were also interpreted using a score plot (Figure 5). Most chemotherapeutic agents showed a positive association with both components 1 and 3, with nonchemotherapeutic agents showing a tendency to be a negatively associated with either the first or the second principal component. Among the antineoplastic drugs, cytotoxic agents (i.e., alkylating agents, antimetabolic agents, antineoplastic antibiotics, platinating agents, and plant-derived alkaloids) were found to be associated with infection following neutropenia. Monoclonal antibodies, which are strongly associated with infection following neutropenia, include drugs that are generally used in combination with cytotoxic agents (e.g., bevacizumab and trastuzumab) and drugs indicated for hematological malignancies (e.g., rituximab). Moreover, the effects of protein kinase inhibitors on neutrophils were plotted separately from cytotoxic agents. The results obtained from this study may help healthcare professionals to appropriately manage drug-induced adverse effects in patients.

\section{Limitations}

This study has three limitations [16,17]. First, although mild adverse effects were only occasionally reported, severe adverse effects were frequently reported, which can lead to reporting bias, a characteristic of self-reporting databases [18]. Second, data obtained from the FAERS database contain blank cells, with some reports having incorrect characters and numbers. Therefore, this study needed to revise the side effects and drug names as much as possible. Third, the cause of the side effects was difficult to determine when multiple drugs were administered [16,17].

\section{Materials and Methods}

\subsection{Database Information}

Since January 2004, the U.S. FDA has continued to add information regarding cases of adverse events associated with all marketed drugs and therapeutic biologic products to the FAERS database [10], which contains adverse events reported by manufacturers to the FDA as required by regulation, along with reports received directly from consumers and healthcare professionals. After downloading the FAERS database, analysis was performed using data reported between April 2004 and September 2020. 


\subsection{Terminology of Analyzed Drugs and Adverse Events}

The analyzed drugs were selected from the FAERS database using the World Health Organization-recommended Anatomical Therapeutic Chemistry (ATC) classification system [19]. "L01: antineoplastic agents" (222 drugs) were extracted from the "ANTINEOPLASTIC AND IMMUNOMODULATING AGENTS" class, one of the 14 major ATC classes.

To analyze CIN, eight preferred terms (i.e., neutropenia (PT code: 10029354), idiopathic neutropenia (PT code: 10051645), granulocytopenia (PT code: 10018687), agranulocytosis (PT code: 10001507), neutropenic sepsis (PT code: 10049151), neutropenic colitis (PT code: 10062959), neutropenic infection (PT code: 10059482), and febrile neutropenia (PT code: 10016288)) were extracted based on MedDRA/J version 23.0.

\subsection{Production of Data Analysis Table}

The FAERS database comprises seven tables related to (a) (DEMO), (b) DRUG, (c) REAC, (d) patient outcomes for the event (OUTC), (e) report sources for the event (RPSR), (f) drug therapy start and end dates for the reported drug (THER), and (g) MedDRA terms coded for the indications (diagnoses) of the reported drugs (INDI). DRUG, REAC, and DEMO tables were analyzed to determine the patient's background and drugs causing the neutropenia (Figure 6).

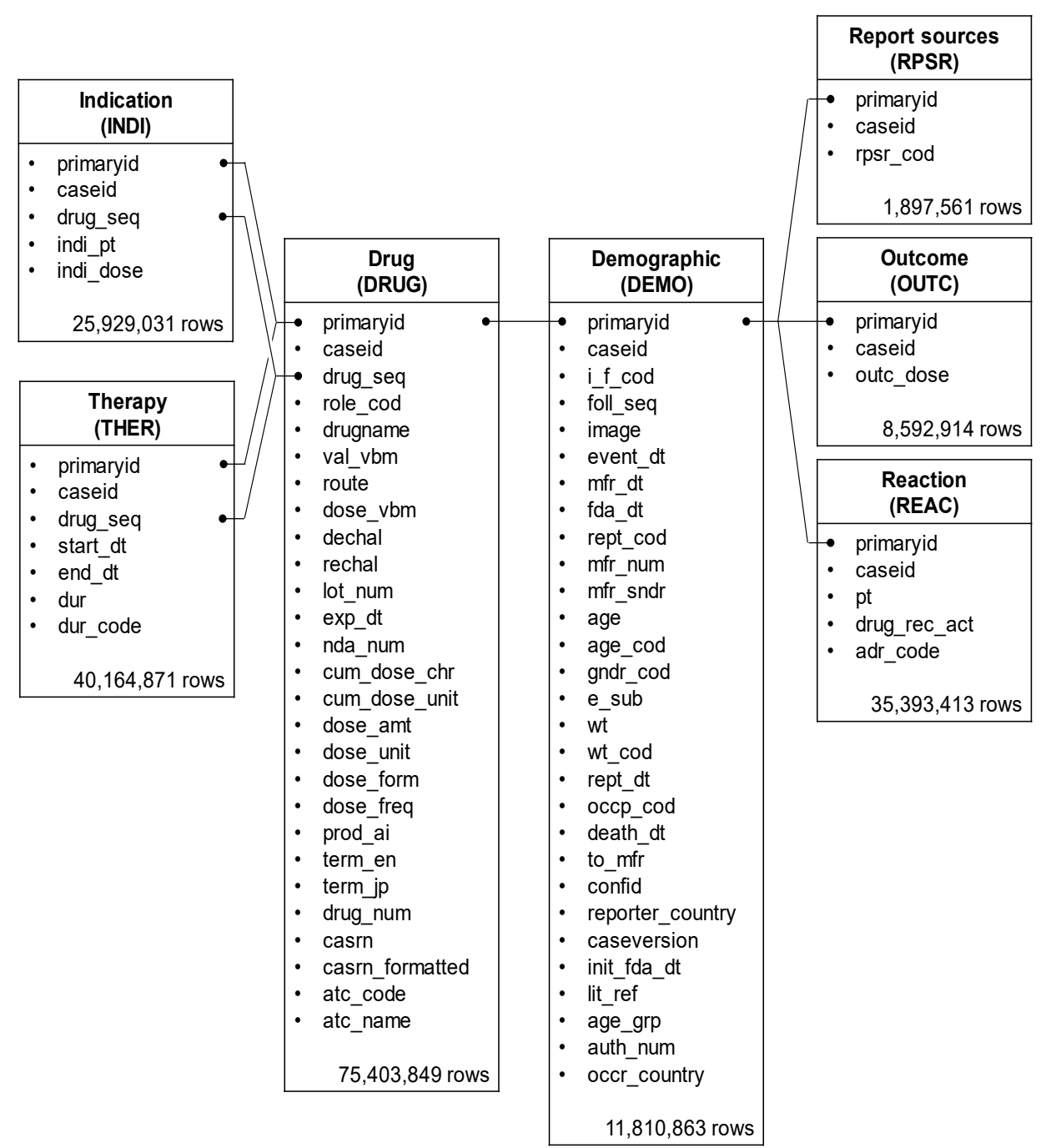

Figure 6. Seven information tables included in the FAERS database. The row number shows the number of reports obtained between April 2004 and September 2020. 
Duplicate data were removed from the DEMO, DRUG, and REAC tables and combined based on the primary ID. Furthermore, only information corresponding to the "primary suspect drug" was extracted from these data and used for constructing the data analysis table (Figure 1). Of this, pediatric patients with cancer, defined as patients with cancer or sarcoma aged $<15$ years, accounted for $0.1 \%$ (2145 patients, 9336 rows).

\subsection{Patient Characteristics Associated with Neutropenia}

Patient characteristics were divided based on the presence and absence of CIN. Age and weight data from the data analysis table were treated as absolute numbers, and $p$ values were calculated using Wilcoxon's rank-sum test. Registered data for a weight of $\geq 150 \mathrm{~kg}$ was assumed to be $150 \mathrm{~kg}$, whereas registered data for an age of $\geq 110$ years was assumed to be 110 years. $p$ values for sex were calculated using Fisher's exact test. Patient factors were analyzed using only datasets that did not include missing values.

\subsection{Univariate Analysis of Relationship between Drugs and Neutropenia}

Reporting odds ratio (ROR) and Fisher's exact test were used to assess the risk of $\mathrm{CIN}$ for each registered drug. Initially, a $2 \times 2$ contingency table of drugs and adverse events were created for each drug based on the information presented in the data analysis table (Table 4). Given that the $2 \times 2$ contingency table could not be calculated with zero cells and that the estimation would become unstable with a small cell frequency, 0.5 was added to all cells as a correction (Haldane Anscombe half correction) [20,21]. In this study, CIN-related drugs were defined as those with a ROR of $\geq 1$ and a Fisher's exact test $p$ value of $<0.05$ [22]. Subsequently, a scatterplot comprising ROR and $p$ values was created for the visual interpretation of adverse drug events. This scatter plot was created by converting ROR to logarithmically transformed odds ratios (lnORs) and the $p$ value obtained from Fisher's exact test to common logarithms ( $-\log 10(p$ value $))$. The scatter plot corresponds to volcano plots frequently used to understand gene expression trends in bioinformatics [23-27].

Table 4. Cross-tabulation and calculation formula for RORs of CIN.

\begin{tabular}{ccc}
\hline Report Type & CIN & Non-CIN \\
\hline Reports with the suspected medicine & a & $\mathrm{c}$ \\
All other reports & $\mathrm{b}$ & $\mathrm{d}$ \\
\hline $\mathrm{ROR}=(\mathrm{a} / \mathrm{b}) /(\mathrm{c} / \mathrm{d})=\mathrm{ad} / \mathrm{bc}$ & &
\end{tabular}

$\operatorname{ROR}=(a / b) /(c / d)=a d / b c$.

\subsection{Cluster Analyses of CIN-Related Drugs}

Among the 185 drugs identified as "L01: ANTINEOPLASTIC AGENTS", 58 (31.4\%) with $\geq 20,000$ reports were analyzed. RORs were calculated from $2 \times 2$ contingency tables for adverse event and registered drugs. Thereafter, the RORs were converted to natural logarithms and used in hierarchical cluster analysis to objectively classify the registered drug with $\geq 20,000$ reports. This analysis used the Ward method based on Euclidean distance with loads from 58 chemotherapeutic agents [12,28].

\subsection{Principal Component Analysis Related to CIN}

The cluster analysis analyzed and roughly classified the antineoplastic agents registered in FAERS (185 drugs, 6.0\%) based on their characteristics. In contrast, the principal component analysis analyzed only antineoplastic agents with $>20,000$ reports (122 drugs, $4.0 \%$ ) among all registered drugs ( 3079 drugs) in the data analysis table and compared more detailed CIN explanatory variable biases. RORs were calculated from $2 \times 2$ contingency tables for adverse events and registered drugs. Thereafter, the RORs were converted to natural logarithms and used in principal component analysis with association matrices [15,29]. The first, second, and third principal components were used to interpret the characteristics of the drugs and their adverse events. 


\subsection{Statistical Analysis}

All analyses were performed using JMP Pro14 (SAS Institute Inc., Cary, NC, USA), with the level of statistical significance being set to 0.05 .

\section{Conclusions}

Our comprehensive analyses of the data in a large dataset in order to compare the risk of CIN as well as the detailed characteristics of patients treated using chemotherapeutic agents highlight the necessity for more careful monitoring of elderly or underweight patients treated with cytotoxic agents, including alkylating agents, antimetabolic agents, antineoplastic antibiotics, platinating agents, and plant-derived alkaloids. Our findings should facilitate the identification of drugs that may cause neutropenia and help healthcare professionals manage drug-induced adverse effects in their patients. Further verification and investigation of the underlying mechanisms in future studies are expected to extensively contribute to the understanding of the CIN risk revealed in our study.

Author Contributions: Conceptualization, Y.U.; methodology, Y.U.; software, Y.U.; validation, M.O., D.K., R.M., T.K. and Y.U.; formal analysis, M.O. and Y.U.; investigation, M.O. and Y.U.; resources, M.O. and Y.U.; data curation, M.O. and Y.U.; writing—original draft preparation, M.O.; writing-review and editing, M.O., D.K., R.M., T.K. and Y.U.; visualization, M.O. and Y.U.; supervision, Y.U.; project administration, Y.U.; All authors have read and agreed to the published version of the manuscript.

Funding: This research received no external funding.

Institutional Review Board Statement: Not applicable.

Informed Consent Statement: Not applicable.

Data Availability Statement: Data is contained within the article.

Conflicts of Interest: The authors declare no conflict of interest.

\section{References}

1. Patel, K.; West, H.J. Febrile neutropenia. JAMA Oncol. 2017, 3, 1751. [CrossRef]

2. Jiang, C.; Hu, X.; Wang, L.; Cheng, H.; Lin, Y.; Pang, Y.; Yuan, W.; Cheng, T.; Wang, J. Excessive proliferation and impaired function of primitive hematopoietic cells in bone marrow due to senescence post chemotherapy in a $\mathrm{T}$ cell acute lymphoblastic leukemia model. J. Transl. Med. 2015, 13, 234-245. [CrossRef]

3. Basu, S.; Hodgson, G.; Katz, M.; Dunn, A.R. Evaluation of role of G-CSF in the production, survival, and release of neutrophils from bone marrow into circulation. Blood 2002, 100, 854-861. [CrossRef]

4. Crawford, J.; Dale, D.C.; Lyman, G.H. Chemotherapy-induced neutropenia; risks, consequences, and new directions for its management. Cancer 2004, 100, 228-237. [CrossRef]

5. Taplitz, R.A.; Kennedy, E.B.; Bow, E.J.; Crews, J.; Gleason, C.; Hawley, D.K.; Langston, A.A.; Nastoupil, L.J.; Rajotte, M.; Rolston, K.; et al. Outpatient management of fever and neutropenia in adults treated for malignancy: American Society of Clinical Oncology and Infectious Diseases Society of America Clinical Practice Guideline Update. J. Clin. Oncol. 2018, 36, 1443-1453. [CrossRef]

6. Kasi, P.M.; Grothey, A. Chemotherapy-induced neutropenia as a prognostic and predictive marker of outcomes in solid-tumor patients. Drugs 2018, 78, 737-745. [CrossRef]

7. Kalinka-Warzocha, E.; Plazas, J.G.; Mineur, L.; Salek, T.; Hendlisz, A.; DeCosta, L.; Vogl, F.D.; Passalacqua, R. Chemotherapy treatment patterns and neutropenia management in gastric cancer. Gastric Cancer 2015, 18, 360-367. [CrossRef]

8. Weycker, D.; Li, X.; Edelsberg, J.; Barron, R.; Kartashov, A.; Xu, H.; Lyman, G.H. Risk and consequences of chemotherapy-induced febrile neutropenia in patients with metastatic solid tumors. J. Oncol. Pract. 2015, 11, 47-54. [CrossRef] [PubMed]

9. Goldman, S.A. Limitations and strengths of spontaneous reports data. Clin. Ther. 1998, 20 (Suppl. C), C40-C44. [CrossRef]

10. FDA Adverse Event Reporting System (FAERS). Available online: https://www.fda.gov/drugs/drug-approvals-and-databases/ fda-adverse-event-reporting-system-faers (accessed on 5 June 2021).

11. Lyman, G.H.; Abella, E.; Pettengell, R. Risk factors for febrile neutropenia among patients with cancer receiving chemotherapy: A systematic review. Crit. Rev. Oncol. Hematol. 2014, 90, 190-199. [CrossRef] [PubMed]

12. Everitt, B.S.; Landau, S.; Leese, M.; Stahl, D. Cluster Analysis, 5th ed.; John Wiley \& Sons: Hoboken, NJ, USA, 2011.

13. Cristofanilli, M.; Turner, N.C.; Bondarenko, I.; Ro, J.; Im, S.A.; Masuda, N.; Colleoni, M.; DeMichele, A.; Loi, S.; Verma, S.; et al. Fulvestrant plus palbociclib versus fulvestrant plus placebo for treatment of hormone-receptor-positive, HER2-negative metastatic breast cancer that progressed on previous endocrine therapy (PALOMA-3): Final analysis of the Multicentre, Double-Blind, phase 3 Randomised Controlled Trial. Lancet Oncol. 2016, 17, 425-439. 
14. HIGHLIGHTS OF PRESCRIBING INFORMATION for IBRANCE. Available online: https://www.accessdata.fda.gov/ drugsatfda_docs/label/2017/207103s004lbl.pdf (accessed on 14 July 2021).

15. Jolliffe, I.T. Principal Component Analysis, 2nd ed.; Springer: New York, NY, USA, 2002.

16. Pariente, A.; Avillach, P.; Salvo, F.; Thiessard, F.; Miremont-Salamé, G.; Fourrier-Reglat, A.; Haramburu, F.; Bégaud, B.; Moore, N. Association Française des Centres Régionaux de Pharmacovigilance (CRPV). Effect of competition bias in safety signal generation: Analysis of a research database of spontaneous reports in France. Drug. Saf. 2012, 35, 855-864. [CrossRef]

17. Avillach, P.; Salvo, F.; Thiessard, F.; Miremont-Salamé, G.; Fourrier-Reglat, A.; Haramburu, F.; Bégaud, B.; Moore, N.; Pariente, A. l'Association des Centres Régionaux de Pharmacovigilance Pilot. Evaluation of an automated method to decrease falsepositive signals induced by co-prescriptions in spontaneous reporting databases. Pharmacoepidemiol. Drug. Saf. 2014, 23, 186-194. [CrossRef] [PubMed]

18. Maeda, R. 5. JADER from pharmacovigilance point of view. Jpn. J. Pharmacoepidemiol. Yakuzai Ekigaku 2014, 19, 51-56. [CrossRef]

19. Lumini, A.; Nanni, L. Convolutional neural networks for ATC classification. Curr. Pharm. Des. 2018, 24, 4007-4012. [CrossRef] [PubMed]

20. Watanabe, H.; Matsushita, Y.; Watanabe, A.; Maeda, T.; Nukui, K.; Ogawa, Y.; Sawa, J.; Maeda, H. Early detection of important safety information. Recent methods for signal detection. Jpn. J. Biomet. 2004, 25, 37-60. [CrossRef]

21. Ohyama, K.; Sugiura, M. Evaluation of the association between topical prostaglandin F2 $\alpha$ analogs and asthma using the JADER database: Comparison with $\beta$-blockers. Yakugaku Zasshi 2018, 138, 559-564. [CrossRef]

22. Van Puijenbroek, E.P.; Bate, A.; Leufkens, H.G.M.; Lindquist, M.; Orre, R.; Egberts, A.C. A Comparison of measures of disproportionality for signal detection in spontaneous reporting systems for adverse drug reactions. Pharmacoepidemiol. Drug. Saf. 2002, 11, 3-10. [CrossRef]

23. Chen, J.J.; Wang, S.J.; Tsai, C.A.; Lin, C.J. Selection of differentially expressed genes in microarray data analysis. Pharm. J. 2007, 7, 212-220. [CrossRef] [PubMed]

24. Hosoya, R.; Uesawa, Y.; Ishii-Nozawa, R.; Kagaya, H. Analysis of factors associated with hiccups based on the Japanese Adverse Drug Event Report Database. PLoS ONE 2017, 12, e0172057. [CrossRef]

25. Toriumi, S.; Kobayashi, A.; Uesawa, Y. Comprehensive study of the risk factors for medication-related osteonecrosis of the jaw based on the Japanese Adverse Drug Event Report Database. Pharmaceuticals 2020, 13, 467. [CrossRef] [PubMed]

26. Okunaka, M.; Kano, D.; Matsui, R.; Kawasaki, T.; Uesawa, Y. Evaluation of the expression profile of irinotecan-induced diarrhea in patients with colorectal cancer. Pharmaceuticals 2021, 14, 377. [CrossRef]

27. Kan, Y.; Nagai, J.; Uesawa, Y. Evaluation of antibiotic-induced taste and smell disorders using the FDA Adverse Event Reporting System Database. Sci. Rep. 2021, 11, 9625. [CrossRef] [PubMed]

28. Umetsu, R.; Abe, J.; Ueda, N.; Kato, Y.; Nakayama, Y.; Kinosada, Y.; Nakamura, M. Adverse event trends associated with over-the-counter drugs: Data mining of the Japanese Adverse Drug Event Report Database. Yakugaku Zasshi 2015, 135, 991-1000. [CrossRef] [PubMed]

29. Nagai, J.; Uesawa, Y.; Shimamura, R.; Kagaya, H. Characterization of the adverse effects induced by acetaminophen and nonsteroidal anti-inflammatory drugs based on the analysis of the Japanese Adverse Drug Event Report Database. Clin. J. Pain. 2017, 33, 667-675. [CrossRef] [PubMed] 